\title{
Bottom Tracking Method Based on LOG/Canny and the Threshold Method for Side-scan Sonar
}

\author{
Shengping Wang ${ }^{1,2}$, Hongtao $\mathrm{Li}^{1,2^{*}}$, Xiaoyu $\mathrm{Li}^{3}$, Jiansong Yang ${ }^{1,2}$ and Quanhong Feng ${ }^{4}$ \\ ${ }^{1}$ College of Surveying and mapping, East China University of Technology, Nanchang 330013, China \\ ${ }^{2}$ Key Laboratory of Watershed Ecology and Geographical Environment Monitoring, NASG, East China University of Technology, \\ Nanchang 330013, China \\ ${ }^{3}$ Guangdong Provincial Channel Surveying and Mapping Center, GuangZhou 510000, China \\ ${ }^{4}$ Multiinfo $3 D$ Laser Scan Solution AB, Stockholm, Sweden
}

Received 6 July 2019; Accepted 22 November 2019

\begin{abstract}
The detection of sea bottom lines is affected by the noise in the water column between the towfish and the seabed. Under these circumstances, using one edge detection technology to detect the bottom line accurately is difficult. A fusion method based on LOG/Canny operator and the threshold approach was proposed in this study to determine sea bottom lines in sonar images accurately. With this method, images filtered by the LOG function and sharpened by a convolution template were utilized as the input images of the Canny detection algorithm to implement bottom tracking. The tracking result was fused with that of the threshold method to obtain merged submarine lines. Interpolation and filtration were subsequently implemented to eliminate outliers. The result was then used to accurately detect sea bottom lines. The measured height and depth were compared using a single-beam depthometer. Results demonstrate that the bottom line is difficult to accurately detect by using only edge detection technologies, such as LOG and Canny, or the threshold method under complex situations in the water column. By contrast, the proposed fusion method can perform detection accurately. Take Mirs Bay of the Shenzhen city as an example, the mean square error of tracking accuracy is determined to be $\pm 0.192 \mathrm{~m}$, which verifies the feasibility of the proposed method. This study provides a reference for the accurate detection of submarine lines in complex conditions wherein large amounts of noise exist in the water column.
\end{abstract}

Keywords: Side-scan sonar, Sea bottom line detection, LOG operator, Canny operator, Threshold method

\section{Introduction}

Side-scan sonar (SSS) is an efficient method to obtain information on seabed topography. The method has been widely used due to its high precision, high efficiency, and high seafloor image quality. SSS is commonly used in marine surveying and mapping, submarine geological exploration, and detection of seabed features [1-5]. In the generated grayscale image, the sea bottom lines, which are the obvious boundaries between the water column and image areas, are composed of a series of submarine points, which is the first real seafloor echo point along the track direction of each ping datum [6]. This line shows the height between the towfish and the sea bottom, and its detection accuracy directly affects the accuracy of slant range correction [7]. Towfish are generally towed during SSS data collection, and the process is affected by waves and their wakes, towfish attitude, self-noise, and environmental noise, all of which pollute the water column area easily. The height of the towfish should satisfy the principles of symmetry and gradual change. According to the principle of symmetry, the left and right transducers of towfish are relatively close to each other, and echoes are simultaneously received from seabed travel. Therefore, the bottom lines extracted from port and starboard sides should be symmetrical to the track line. The principle of gradual change indicates that towfish

*E-mail address: ta02218@live.com

ISSN: $1791-2377$ @ 2019 School of Science, IHU. All rights reserved.

doi:10.25103/jestr.126.17 height, which is influenced by seabed topography and towfish depth, exhibits gradual change. If the height of the towfish is uncorrected, then sonar image processing will be affected [8-10]. At present, the bottom line is usually extracted by using a semi-manual approach during data postprocessing instead of performing bottom tracking operations during data collection on site, which is inefficient and largely subjective. Furthermore, the bottom line in an area with large noise can be extracted with high precision. Detecting the bottom line and determining the height of the towfish effectively are urgent problems to be solved.

On the basis of the basic technology of digital image processing, this study introduces a method of combining the LOG/Canny operator and the threshold method to detect the sea bottom line in an SSS image. The developed technique is compared with existing methods for tracking the sea bottom line in terms of efficiency and accuracy. The aim is to locate the bottom point under complex circumstances accurately. This work provides a reference for the accurate tracking of submarine lines.

\section{State of the Art}

SSS data processing involves data decoding, sea bottom line detection, distortion correction, geocoding, image mosaic, target segmentation, and target recognition [7,11-14]. Many commercial data processing software, such as Triton, SonarWeb, and Discovery, use a sea bottom line detection 
module in the threshold method but exhibit low precision in complex cases [15]. Studies on algorithms for sea bottom line detection are rare. Maximum amplitude and gradient methods are commonly used approaches [6]. Xu [7] realized bottom line detection by using the traditional threshold method with minimal noise in the water column. Zhang [6] used the LOG operator of edge detection to extract the edge of the image and achieve automatic detection of the sea bottom line. However, the LOG operator has limitations. It can only eliminate grayscale variation at a scale that is less than variance. Furthermore, when the influence of self-noise and environmental noise is too large, the LOG operator mistakenly retains some image noise as edges, resulting in a false extraction of the sea bottom line. Galdrán et al. [10] performed a similarity analysis among the corresponding regions extracted from a sonar image and indicated that MIRA has excellent normalization performance. Lubis et al. [14] used the fuzzy C-means clustering algorithm to divide all pixels in an SSS image into a set of C fuzzy clusters, thus segmenting the shadow region. Zhao et al. $[16,17]$ proposed a comprehensive method that integrates the traditional threshold, last peak, and anomaly tracking segment repair method of terrain change. The authors achieved automatic extraction of the sea bottom line, but the implementation of the developed method was complicated. Al-Rawi et al. [18] detected the first submarine echo point via smoothing cubic spline regression and moving average filter, but they did not apply their method to sonar images with complex noise in the water column region. Manik et al. [19] obtained sounding and side-scan data simultaneously and used PDS 2000 and SonarWeb to process them separately. Although the authors obtained a good seabed height, they did not provide the corresponding detection methods. Tegowsk et al. [20] estimated the height of the seabed and seabed features by using 2D discrete wavelet decomposition coefficients of sonar images as the input of the self-organizing neural network classification algorithm. Ji-Bo et al. [25] introduced a new LOG operator-based seabed tracking method for image edge detection. The method can be used in accurate seabed tracking and to correct slant range in SSS data. Actual data processing results showed that this method has obvious advantages compared with the traditional tracking method. Chavez et al. [26] posited that the bottom line is the obvious boundary line on both sides of the water column area, which indicates that the height from the lateral sweeping sound to the seabed is an important parameter for target measurement, slant range correction, and image gray level equalization. Berkson et al. [27] indicated that accurate detection of the bottom line is the key to the subsequent correction of the slant range.

The results of these studies were obtained through a single edge detection technology or a fusion of multiple methods. However, edge detection technology fails when complex conditions exist in the water column, and the fusion of multiple methods is highly complex. The threshold method is also affected by experiences with low efficiency and precision. For these reasons, we propose a new method to extract the seabed line by using Canny edge detection after LOG filtering. The result is fused with that of threshold detection. The proposed method provides a certain reference for the accurate tracking of seabed lines under complex circumstances.

The remainder of this study is organized as follows. Section 3 describes the process of LOG/Canny edge detection and compares this technology with other edge detection technologies. The threshold detection method is described through the difference and normalization of the original data, and the steps of the fusion method and experimental process are presented. Section 4 compares the results of the three methods and shows that the experimental results of the fusion method are better than those of the other methods. The last section summarizes the study and presents relevant conclusions.

\section{Methodology}

\subsection{LOG/Canny edge detection}

The edge of an image refers to the collection of pixel points where the grayscale of the surrounding pixels sharply changes [21, 22]. Owing to the special procedure of generating SSS images, namely, filling an image with noise, Gaussian noise with a normal distribution can be removed by using a common filtering method known as Gaussian Laplacian filter.

\subsubsection{LOG function filter}

Suppose that the raw image is $f(\mathrm{x}, \mathrm{y})$ and the Gaussian function is $G(\mathrm{x}, \mathrm{y})$. First, $G(\mathrm{x}, \mathrm{y})$ is used as a low-pass filter to smooth the image. Second, the second-order differential operation is adopted to extract edges from the filtered image. This process is equivalent to the application of the secondorder differential operation to the Gaussian function. Third, the convolution operation is applied to the raw image by using the second-order differential result of the Gaussian function. Lastly, the $\operatorname{LOG}(\mathrm{x}, \mathrm{y})$ function is obtained as follows:

$$
\operatorname{LOG}(\mathrm{x}, \mathrm{y})=\frac{1}{\pi \sigma^{4}}\left(\frac{x^{2}+y^{2}}{2 \sigma^{2}}-1\right) \exp \left(-\frac{x^{2}+y^{2}}{2 \sigma^{2}}\right)
$$

The experiment shows that noise can be effectively suppressed when the LOG operator is used for edge detection. The grayscale value of the image is less than that of the LOG function, which is composed of noise. This study applies this principle to image filtering and noise reduction [21].

$$
f(\mathrm{x}, \mathrm{y})<\operatorname{LOG}(\mathrm{x}, \mathrm{y})
$$

where $f(\mathrm{x}, \mathrm{y})$ represents the grayscale value of the raw image and $\operatorname{LOG}(\mathrm{x}, \mathrm{y})$ represents the value of the LOG function. The image value that satisfies the formula can be identified as noise. The influence of noise, interference of the subsequent edge detection, and strength of the image edge are eliminated after image filtering. Hence, image sharpening is required.

\subsubsection{Image sharpening}

The image is sharpened after filtration, and a second-order differential template is employed to convolve with the filtered image. The template is as follows:

$$
\left[\begin{array}{ccc}
0 & -1 & 0 \\
-1 & 4 & -1 \\
0 & -1 & 0
\end{array}\right]
$$

The sharpened image can be utilized as an input image 
for the Canny detection process.

\subsubsection{Canny operator detection}

The Canny edge detection operator is popular and demonstrates excellent performance. Although it is a firstorder differential operator, it extends the traditional firstorder operator by adopting non-maximal suppression and a double-threshold test. The use of non-maximal suppression can effectively suppress the multi-edge response and improve edge location accuracy. The use of the dualthreshold test can effectively reduce the missed detection rate $[26,27]$.

Calculate the gradient amplitude and direction angle

The traditional Canny operator uses a $2 \times 2$ template gradient operator to calculate gradient amplitudes $G_{x}$ and $G_{y}$ of the smoothed image in x and y directions. On this basis, this study uses the Sobel first-order differential template and image convolution to obtain the gradient amplitudes. The Sobel template is expressed as

$$
G_{x}=\left(\begin{array}{ccc}
-1 & 0 & 1 \\
-2 & 0 & 2 \\
-1 & 0 & 1
\end{array}\right) \quad G_{y}=\left(\begin{array}{ccc}
-1 & -2 & -1 \\
0 & 0 & 0 \\
1 & 2 & 1
\end{array}\right)
$$

Gradient amplitude: $G(\mathrm{x}, \mathrm{y})=\sqrt{G_{x}^{2}+G_{y}^{2}}$

Gradient direction angle: $\theta=\arctan \left(\mathrm{G}_{y} / \mathrm{G}_{x}\right)$

During the calculation of the gradient direction angle, it is merged into four directions, namely, $0^{\circ}, 45^{\circ}, 90^{\circ}, 135^{\circ}$. For all edges, let $0^{\circ}=180^{\circ}, 45^{\circ}=225^{\circ}$. Thus, $\left[-22.5^{\circ} \sim 22.5^{\circ}\right]$ and $\left[157.5^{\circ} \sim 202.5^{\circ}\right]$ are merged into $0^{\circ}$. The other angles are subjected to the same process.

\section{Non-maximal suppression}

After determining the direction angle, the gradient amplitude in the eight vicinities of the pixel $(\mathrm{i}, \mathrm{j})$ is checked in accordance with the obtained gradient direction angle.

- If $\theta(i, j)=0^{\circ}$, then cells $(i+1, j),(i, j)$, and $(i-1, j)$ are compared.

- If $\theta(\mathrm{i}, \mathrm{j})=45^{\circ}$, then cells $(\mathrm{i}+1, \mathrm{j}+1),(\mathrm{i}, \mathrm{j})$, and $(\mathrm{i}-1, \mathrm{j}-1)$ are compared.

- If $\theta(i, j)=90^{\circ}$, then cells $(i, j+1),(i, j)$, and $(i, j-1)$ are compared.

- If $\theta(\mathrm{i}, \mathrm{j})=135^{\circ}$, then cells $(\mathrm{i}+1, \mathrm{j}-1),(\mathrm{i}, \mathrm{j})$, and $(\mathrm{i}-1, \mathrm{j}+1)$ are compared.

The gradient amplitudes of the three pixels are compared. If the gradient amplitude of $(i, j)$ is larger than that of the two other points, then $(i, j)$ will be considered the center point of the edge to be recorded; otherwise, it will be deleted.

\subsubsection{Flow of the LOG/Canny algorithm}

Analysis of the advantages and disadvantages of five edge detection algorithms, namely, Laplace, Roberts, Kirsch, Canny, and LOG, verifies that only the edge detection algorithm can track the bottom line effectively when the noise in the water column area is weak. The combination of LOG/Canny, which is investigated in this study, performs well. The algorithm flow chart of LOG/Canny shown in Figure 1, comparison of experimental is showed in Figure 2.

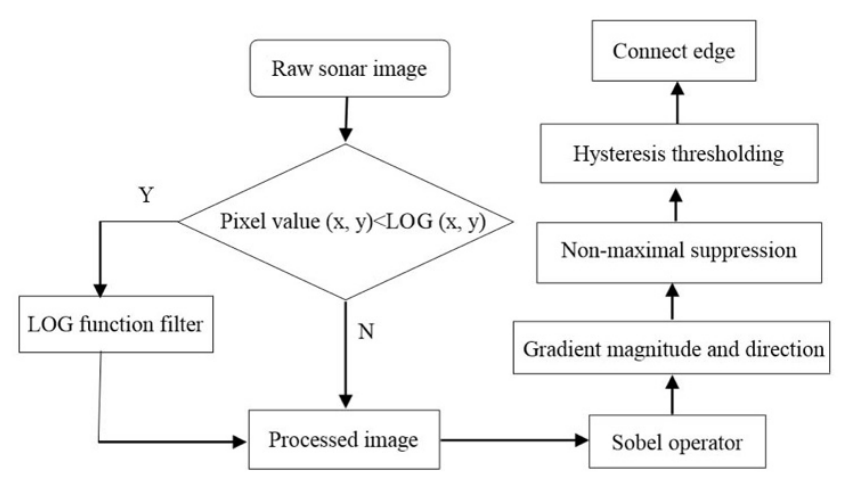

Fig. 1. LOG/Canny algorithm flow chart

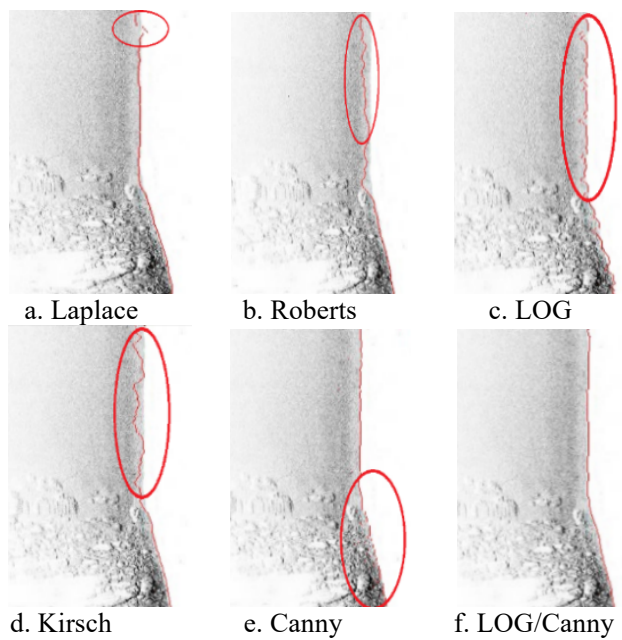

Fig. 2. Comparison of the experimental results of each algorithm

Table 1. Comparison of the time consumption of the methods

\begin{tabular}{c|c|c|c|c|c|c}
\hline Methods & LOG/Canny & LOG & Canny & Roberts & Laplace & Kirsch \\
\hline Time (s) & 0.5682 & 0.3976 & 0.413 & 1.013 & 0.2978 & 0.4736 \\
\hline
\end{tabular}

The time required by each method is recorded in Table 1 . The method studied in this work can obtain the result in approximately $0.5 \mathrm{~s}$. The method's accuracy and efficiency in submarine line detection are verified. Although the other methods consume less time, they cannot guarantee the accuracy of submarine line detection.

Figures $2 \mathrm{a}$ to $2 \mathrm{e}$ show comparisons. The detection effects of Kirsch, Roberts, Laplace, LOG, and Canny increase successively. The Kirsch operator is more complex than the other methods. It uses eight templates to perform convolution with the image to obtain gradients. The eight templates represent eight directions and achieve the maximum response to eight specific edge directions in the image. The maximum value is regarded as the edge point of the image. The Roberts operator is simpler than the other methods because the edge is searched by the local difference operator. The edge is detected by using the difference between two adjacent pixels in the diagonal direction to approximate the gradient amplitude. The effect of detecting the vertical edge is better than that of detecting the oblique 
edge, which has high positioning accuracy but is sensitive to noise. The Laplace algorithm uses the Laplacian template to convolve with the image then performs a threshold test. Figure 2c shows the effect of the sea bottom line tracked with the method described in [6]. Figure 2e shows the effect of the Canny detection operator, which is ideal. These methods cannot effectively identify the weak echo from the sea bottom line and involve threshold selection. The rationality of threshold selection affects the accuracy of edge location. Reducing human intervention is crucial regardless of whether an adaptive threshold can be obtained or not.

Figure $2 \mathrm{f}$ shows the LOG/Canny method used in this study. This method presents obvious advantages over the other methods. The experimental results show that this method can effectively detect the sea bottom line with a weak echo and the water column area with minimal noise. During the calculation of the gradient amplitude, the Sobel first-order differential template is applied instead of the traditional Canny gradient operator. Hence, an improved result is obtained. During the calculation of the image gradient direction angle, this study merges the gradient direction angle into four directions. Accordingly, the efficiency of the calculation is enhanced, but the detection accuracy is diminished. Linear interpolation can be used to calculate gradient values in all directions and improve detection accuracy.

\subsection{Threshold method}

The image edge detection algorithm can perform accurate tracking of the sea bottom line when the noise in the water column area is weak or the area is substantially noiseless, as shown in Figure 2. However, SSS often suffers from noise, such as the interference of the beam side lobe, surface echo and tail flow, and suspension, when collecting data. Figure 3 shows these features. The single image edge detection algorithm can no longer perform accurate tracking of the sea bottom line in the sonar images with a large amount of noise in the water column area. The algorithm needs to be combined with other methods to meet the requirements.

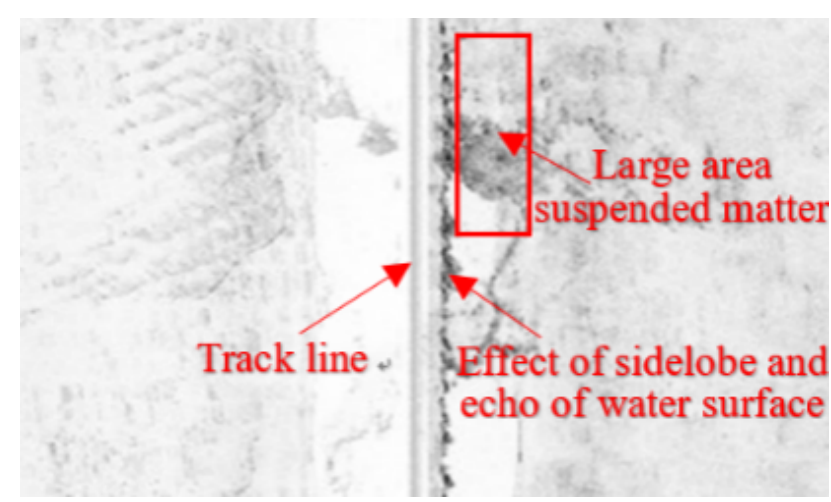

Fig. 3. Effect of complex environmental noise on the water column area

The threshold method is easy to implement. First, the first-order difference, which is the difference between two consecutive items in the discrete function, is applied to raw data. Given $\mathrm{X}(\mathrm{k}), \mathrm{Y}(\mathrm{k})=\mathrm{X}(\mathrm{k}+1)-\mathrm{X}(\mathrm{k})$ is the first-order difference of this function. The difference effect is shown in Figure 4. Second, the difference result is normalized to the range of $[0,1]$ because the range of raw data with the difference is too large. The fluctuation range of threshold selection is also large, thereby narrowing the data interval to simplify threshold selection. During data processing, data are made sparse, and only the data within a certain interval close to the water column area are used in the calculation. The method can easily determine the location of the mutation point (arrows in Figure 4). The initial threshold can be selected artificially by randomly selecting one ping datum. Several sections may have different thresholds. Thus, manual adjustment is necessary. Continuous points are set between 3 and 7 in accordance with the water column area noise. The value in this work is 6 . Specifically, if the grayscale values of six consecutive sample points satisfy the above-mentioned conditions, then the first sample point is considered the seafloor point. The result of using the threshold method is shown in Figure 5. The port point should be placed as far left as possible to reduce the water column area, and the starboard point should be placed as far right as possible. The order is port (right to left) then starboard (left to right).

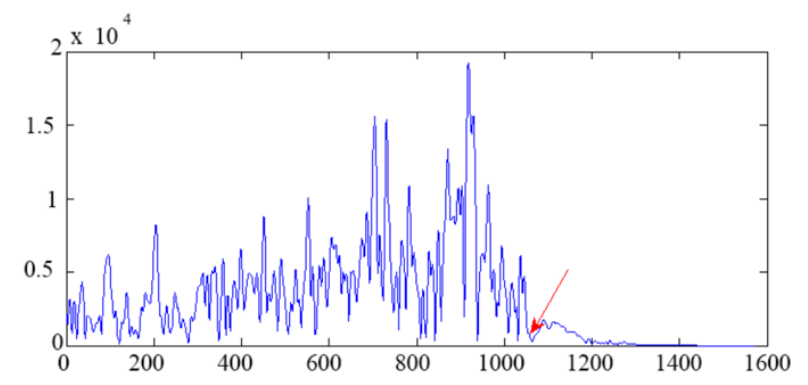

(a)

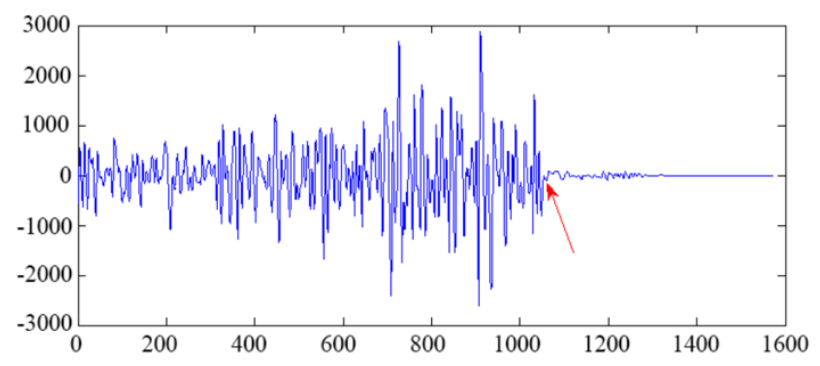

(b)

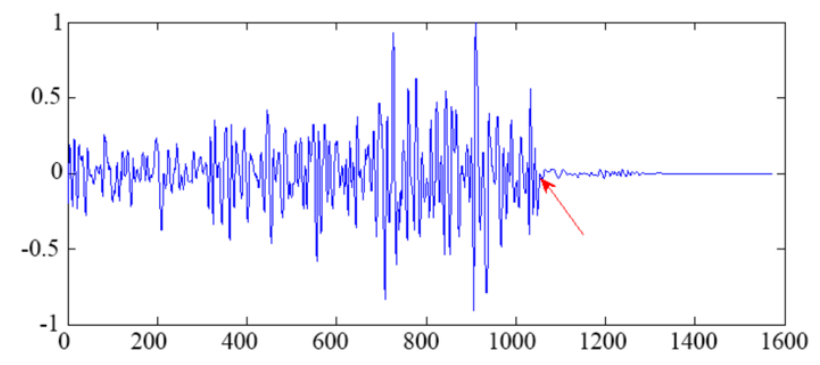

(c)

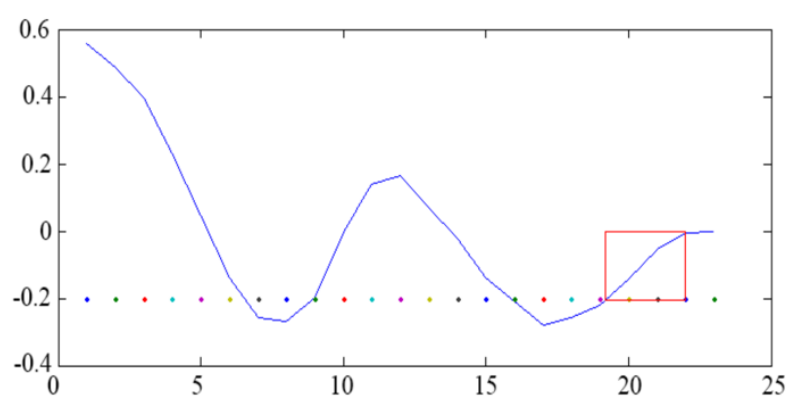

(d)

Fig. 4. Port ping data (a), first-order difference (b), normalization (c), and partial amplification (d) 
The threshold detection results show a large deviation on the side with plenty of noise in the water column area. The side with less noise can be effectively tracked. The single threshold method cannot achieve the ideal detection effect.
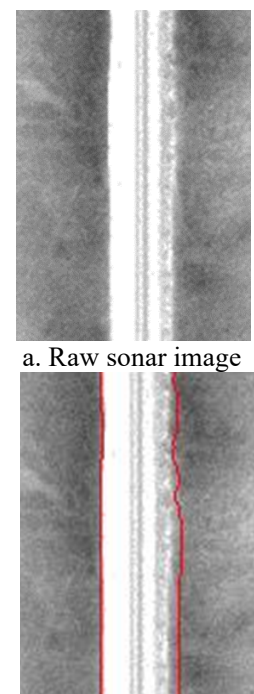

b. Result of the threshold method

Fig. 5. Tracking the sea bottom line by using the threshold method

\subsection{Fusion method experiment process}

Step 1) Sound intensity is transformed to grayscale. XTF data are decoded to generate a grayscale file.

Step 2) The image is loaded. A new bitmap variable is created, and a complete single-band sonar image $f(\mathrm{x}, \mathrm{y})$ is loaded.

Step 3) The image is filtered. $\operatorname{LOG}(\mathrm{x}, \mathrm{y})$ is calculated with Equation (2) to filter and denoise the original sonar image $f(\mathrm{x}, \mathrm{y})$ and obtain image $f^{\prime}(\mathrm{x}, \mathrm{y})$, in which image Gaussian noise is eliminated.

Step 4) The image is sharpened. During the filtering process, the edge strength of the image is reduced by performing image sharpening after Step 3 to obtain image $f^{\prime \prime}(\mathrm{x}, \mathrm{y})$.

Step 5) The gradient amplitude and direction angle are calculated using Equations (3) and (4). The horizontal and vertical gradient amplitudes are calculated using Sobel's first-order differential template. The orientation angles are approximately classified.

Step 6) Non-maximal suppression is performed on the image according to the classified direction angle. An edge gradient amplitude histogram is generated, the double threshold is selected, and the edges are connected to obtain sea bottom line 1.

Step 7) Threshold detection is performed for each ping datum, and the detected sea bottom points are connected, filtered, and smoothed to obtain sea bottom line 2 .

Step 8) Sea bottom lines 1 and 2 are fused. Fusion is the average of the two sea bottom points corresponding to lines 1 and 2. The judgment principle after fusion is whether the principles of symmetry and gradual change of the towfish are satisfied. If so, then the result is the final sea bottom point; otherwise, the current sea bottom point is interpolated and smoothed. The interpolation uses a simple local linear interpolation. Smoothing involves averaging the horizontal coordinates of the five upper and lower ping points.

Step 9) Some ping data at the edge of the image are interpolated to obtain the tracked image of the final sea bottom line.

The step1-9 of this method flow is showed in Figure 6.

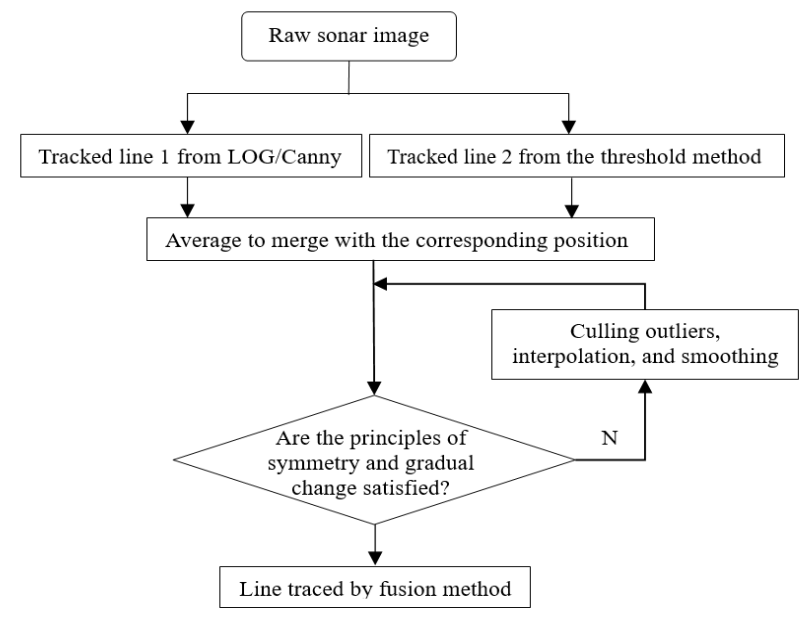

Fig. 6. Fusion method flow chart for tracking the sea bottom line

\section{Result analysis and discussion}

The survey data of Mirs Bay in the Shenzhen city were utilized in this experiment, and dual-frequency SSS equipment was used. Two data groups with high frequency (high resolution, sweep width of $2 \times 75 \mathrm{~m}$ ) and low frequency (ordinary resolution, sweep width of $2 \times 200 \mathrm{~m}$ ) were collected. The results of the adjacent track line overlapped by $100 \%$, and surveying was synchronized with single beam sounding. A total of 171 lines were completed. The SSS instrument is Edgetech 4200FS, and its frequency is 385 KHz. The single beam echo sounder is HY1600. The survey area is shown in Figure 7. The depth of the survey area changes from $5 \mathrm{~m}$ to $15 \mathrm{~m}$. Few geomorphological forms are present in the survey area, but they make up a large number of sand spots, fishing nets, bunkers, anchor trench (anchor marks), reefs, and so on.

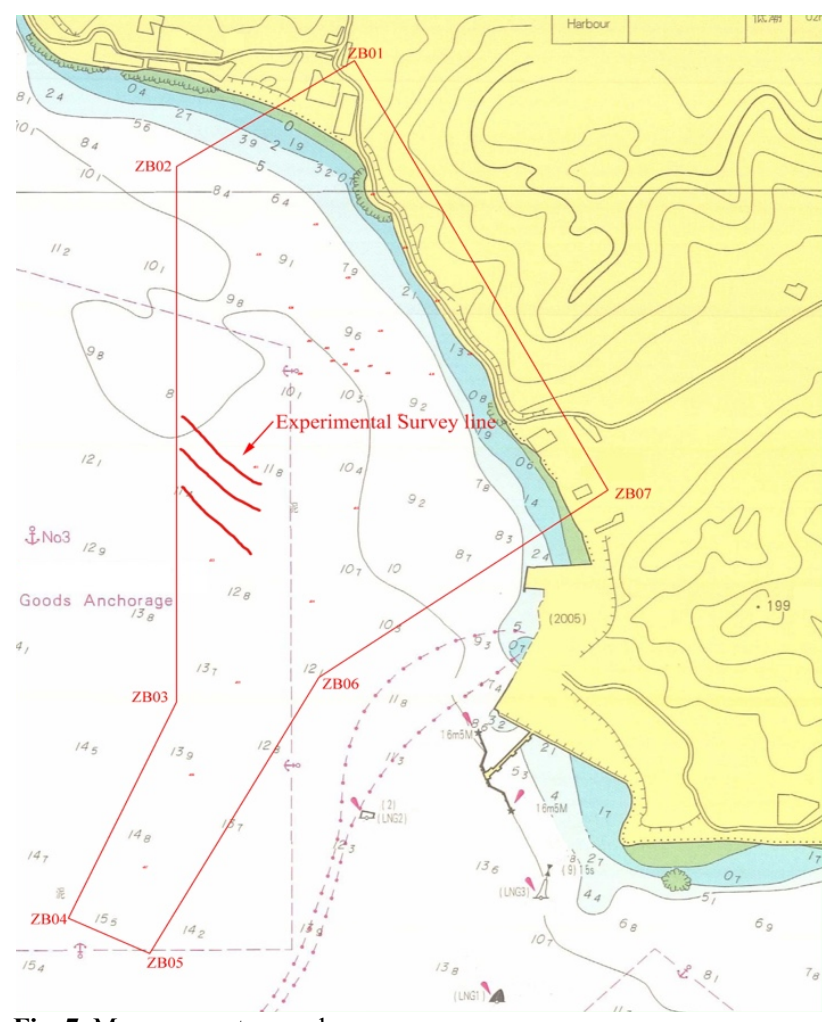

Fig. 7. Measurement area plane 

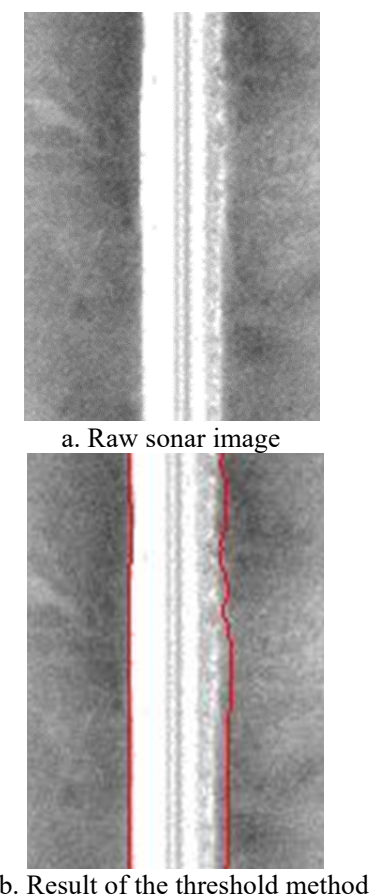

Fig. 8. Comparison of the results of the three methods

Table 2. Comparison of the time consumption of the three methods

\begin{tabular}{c|c|l|l}
\hline Methods & LOG/Canny & $\begin{array}{c}\text { Threshold } \\
\text { Method }\end{array}$ & $\begin{array}{c}\text { Fusion } \\
\text { Method }\end{array}$ \\
\hline Time (s) & 0.567 & 0.354 & 0.682 \\
\hline
\end{tabular}

The LOG/Canny, threshold, and fusion methods are used to track the sea bottom line in a sonar image with substantial noise in the water column area. The experimental results are shown in Figure 8, and the running time of each method is shown in Table 2. From the perspective of time, the run demand is basically satisfied. The tracking results prove that the LOG/Canny and threshold methods cannot effectively detect the sea bottom line in the sonar image in a complex water column environment. By contrast, the fusion method produces optimal tracking results and has an ideal detection time.

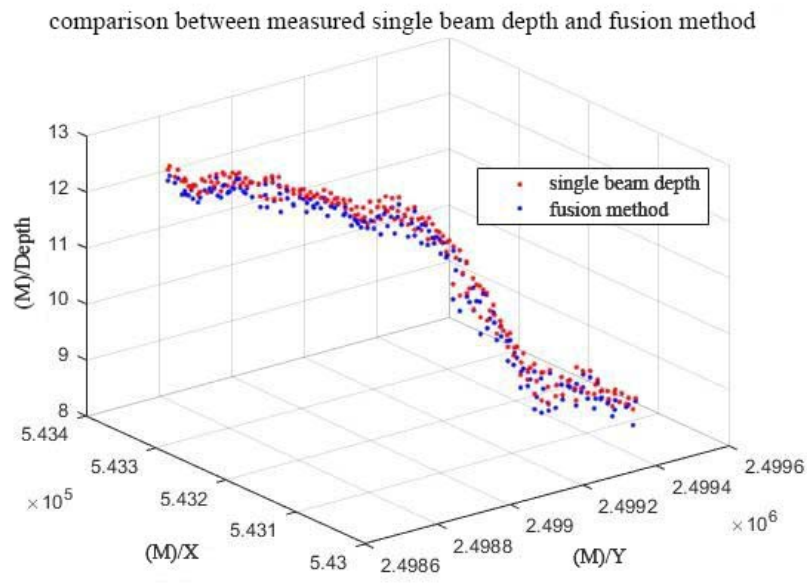

(a) Comparison of measured single beam bathymetric values with the experimental results in this study

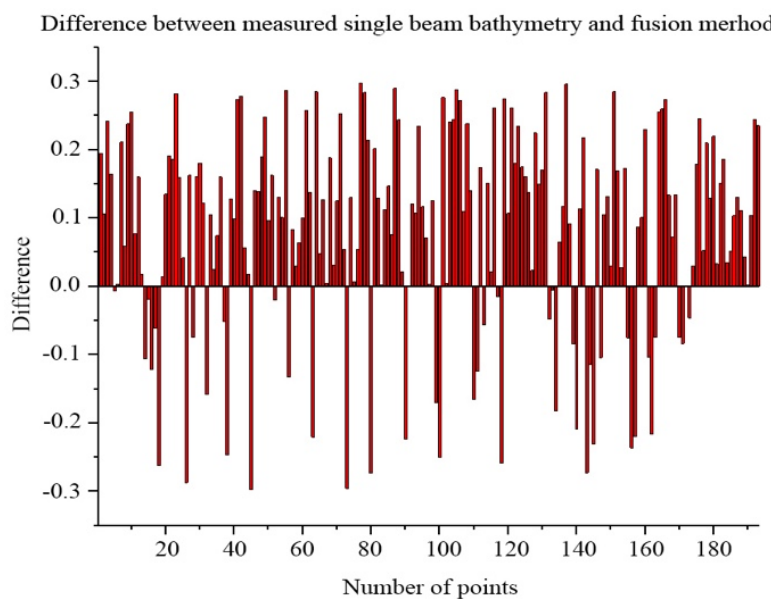

(b) Difference between single beam bathymetry values and the experimental results in this study

Fig. 9. Comparison of single beam bathymetric values with the experimental results and their differences

Table 3. Statistical results of single beam bands and fusion methods

\begin{tabular}{l|c|c|c|c}
\hline \multicolumn{1}{c|}{ Strip } & $\begin{array}{c}\text { Sea } \\
\text { bottom's } \\
\text { height } \\
\text { Max/M }\end{array}$ & $\begin{array}{c}\text { Sea } \\
\text { bottom's } \\
\text { height } \\
\text { Min/M }\end{array}$ & Average/M & $\begin{array}{c}\text { Root } \\
\text { mean } \\
\text { square } \\
\text { error/ } \pm \mathbf{M}\end{array}$ \\
\hline $\begin{array}{l}\text { S1 } \\
\text { Single } \\
\text { beam S1 }\end{array}$ & 12.98 & 8.80 & 11.10 & 0.160 \\
\hline $\begin{array}{l}\text { S2 } \\
\text { Single } \\
\text { beam S2 }\end{array}$ & 13.30 & 8.98 & 11.08 & 0.185 \\
\hline $\begin{array}{l}\text { S3 } \\
\text { Single } \\
\text { beam S3 }\end{array}$ & 10.65 & 7.32 & 9.10 & 0.233 \\
\hline
\end{tabular}
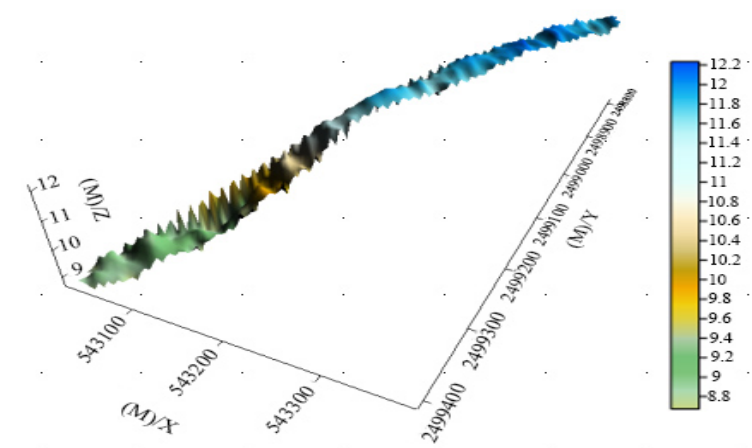

(a)

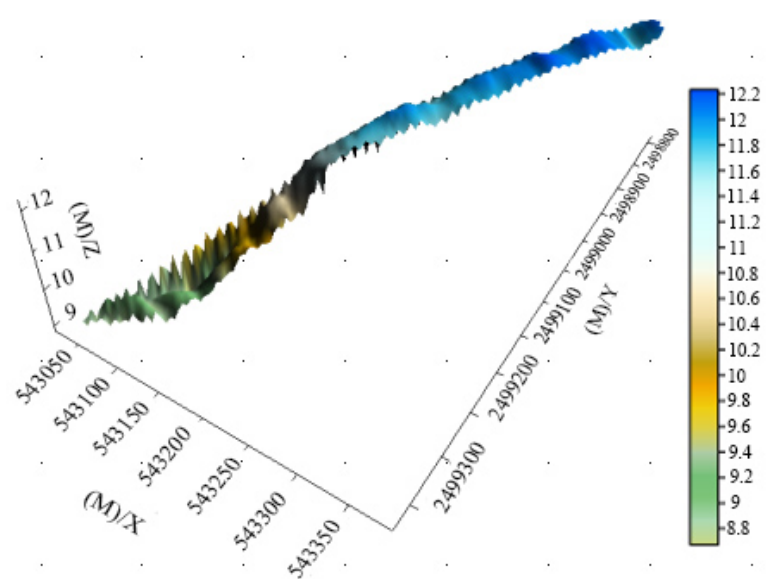

(b)

Fig. 10. DEM of the single beam (a) and DEM of the detected sea 
bottom line (b)

When the water column area has considerable noise, the single image edge detection algorithm and threshold method cannot effectively track the sea bottom line, as illustrated in Figure 8. Therefore, a fusion method is proposed in this study to solve the problem of accurately tracking the sea bottom line in the case of considerable noise in the water column area. To quantitatively determine the accuracy of the fusion method for detecting the sea bottom line, information extracted from the SSS strip image is used to calculate the seabed topographic elevation. The SSS strip tracking point is down-sampled because the frequency of SSS much larger than the single beam sounding frequency. Then, the single beam depth value of the SSS strip is interpolated using the nearest single beam depth value by the nearest neighbor interpolation. DEM is compared between the topographic elevation corresponding to the sea bottom line obtained by the fusion method and the single-beam depth value of SSS strips after interpolation. The comparisons of water depth and its difference are shown in Figures 9 and 10. The two terrains show consistent variability. Figure $9 \mathrm{~b}$ presents comparisons of the depth values detected by the fusion method and measured by the single beam method. The depth difference detected by the fusion method is basically $0.3 \mathrm{~m}$ or less. Thus, the effect is good. Table 3 indicates that the statistical results of the system deviations for strips S1, S2, and $\mathrm{S} 3$ are $\pm 0.16, \pm 0.185$, and $\pm 0.233 \mathrm{~m}$, respectively. These results are consistent with the actual depth-sounding accuracy indicators, thereby demonstrating the effectiveness of the fusion method for tracking the sea bottom line.

\section{Conclusions}

To detect the seafloor accurately in sonar images under complex water environments, this study compared several common image edge detection algorithms. The traditional Canny gradient operator was modified to address the shortcomings of existing detection operators. LOG and Canny were combined for detection and then fused with the threshold method. Improved results were achieved. The following conclusions were obtained:

(a) When minimal noise is present in the water column region, edge detection technology can be applied to the extraction of the submarine line.

(b) When a large amount of noise exists in the water column region, various methods must be combined to extract the seafloor effectively. Using a single method in this case produces a large error.

The newly proposed fusion method that combines edge detection and the threshold method effectively solves the problems above. Thus, this work has a certain reference value for practical application. However, seafloor detection of SSS under complex conditions remains a subject to be studied. Accurately detecting the seafloor height is important for subsequent slant range correction, image mosaic, and target recognition.

\section{Acknowledgements}

This work was supported by the Open Fund Project of Jiangxi Provincial Digital Land and Environment Laboratory (DLLJ201813), National Natural Science Foundation of China (41206078 and 41806114), Natural Science Foundation of Jiangxi Province in China (20181BAB216031), and Jiangsu Province Surveying and Mapping Geographic Information Research Project (JSCHKY201915).

This is an Open Access article distributed under the terms of the Creative Commons Attribution License

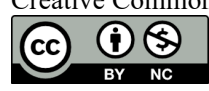

\section{Reference}

1. J Xu, SP Wang., "Research on refinement generation technology of side scan sonar image ". China Water Transport (monthly), 16(9), 2016, pp.283-286.

2. Bryant R., "Side Scan Sonar for Hydrography-An Evaluation by the Canadian Hydrographic Service". The International Hydrographic Review, 52(1), 2015, pp.43-55.

3. Nakamura K, Toki T, Mochizuki N., "Discovery of a new hydrothermal vent based on an underwater, high-resolution geophysical survey". Deep Sea Research Part I: Oceanographic Research Papers, 74, 2013, pp.1-10.

4. Healy C A, Schultz J J, Parker K., "Detecting Submerged Bodies: Controlled Research Using Side-Scan Sonar to Detect Submerged Proxy Cadavers". Journal of Forensic Sciences. 60(3), 2015, pp.743-752.

5. Davy C M, Fenton M B., "Technical Note: Technical note: sidescan sonar enables rapid detection of aquatic reptiles in turbid lotic systems". European Journal of Wildlife Research, 59(1), 2013, pp.123-127.

6. Zhang J B, Pan G F, Ding W F., "Bottom track method in side-scan sonar data processing based on LOG operator". Marine Science Bulletin, 29(3), 2010, pp.324-328.

7. J Xu., "Research on Key Technology of Side Scan Sonar Image Mosaic and Segmentation". Master thesis of East China University of Technology, China, 2017, pp.30-38.

8. XQ Deng, DC Gong, R Luo., "Research on geocoding technology of side scan sonar image". Hydrographic Surveying and Charting. (4), 2002, pp.14-17.
9. TENG HZ, YAN XM, LI SQ., "Processing Techniques of Enhancement for Side Scan Sonar Images". Hydrographic Surveying and Charting, (2), 2004, pp.47-49.

10. Al-Rawi M S, Galdrán A, Yuan X., "Intensity normalization of sidescan sonar imagery". In: International Conference on Image Processing Theory TOOLS and Applications. Paris, France: IEEE, 2017, pp.1-6.

11. Huo G, Yang S X, Li Q., "A Robust and Fast Method for Sidescan Sonar Image Segmentation Using Nonlocal Despeckling and Active Contour Model”. IEEE Transactions on Cybernetics, 47(4), 2017, pp.855-872.

12. Klaucke I., "Submarine Geomorphology". Cham: Springer-Cham, Switzerland, 2018, pp.13-24.

13. Anitha U, Malarkkan S., "Proceedings of the International Conference on Data Engineering and Communication Technology". Singapore: Springer-Singapore, Singapore, 2017, pp.363-370.

14. Lubis M Z, Kausarian H, Anurogo W., "Seabed Detection Using Application of Image Side Scan Sonar Instrument (Acoustic Signal)". Journal of Geoscience, Engineering, Environment, and Technology, 2(3), 2017, pp.230-234.

15. Berkson J M, Clay C., "Transformation of Side-Scan Sonar Records to a Linear Display". The International Hydrographic Review, 50(2), 2015, pp.55-59.

16. Zhao J, Wang X, Zhang H., "An Automatic Bottom Extracting Method for Side-Scan Sonar Image". Geomatics \& Information Science of Wuhan University, 42(12), 2017, pp.1797-1803. 
Shengping Wang, Hongtao Li, Xiaoyu Li, Jiansong Yang and Quanhong Feng/

Journal of Engineering Science and Technology Review 12 (6) (2019) 134 - 141

17. Zhao J, Wang X, Zhang H., "A Comprehensive Bottom-Tracking Method for Sidescan Sonar Image Influenced by Complicated Measuring Environment". IEEE Journal of Oceanic Engineering, PP(99), 2016, pp.1-13.

18. Al-Rawi M, Elmgren F, Frasheri M., "Algorithms for the detection of first bottom returns and objects in the water column in sidescan sonar images" In: OCEANS '17 A Vision for our Marine Future OCEANS '17, Aberdeen, United Kingdom: IEEE, 2017, pp.1-5.

19. Manik H M, Hartoyo D, Rohman S., "Underwater Multiple Objects Detection and Tracking using Multibeam and Side Scan Sonar". International Journal of Applied Information Systems, 7(4), 2014, pp. 5 -8.

20. Tęgowski J, Zielinski A., "Synthesis and wavelet analysis of sidescan sonar sea bottom imagery". Polskie Towarzystwo Akustyczne Oddziat Gdański, 9, 2006, pp.199-208.

21. He Q, Yan li., "Algorithm of Edge Detection Based on LOG and Canny Operator”, Computer Engineering, 37(03), 2011, pp.210-212.
22. LM Guan, L Li, J Lin., "Image edge detection method based on improved LOG operator". Mechanical \& electrical engineering magazine, 27(12), 2010, pp.113-115.

23. Xu HK, Qin YY, Chen HR., "An Improved Algorithm for Edge Detection Based on Canny". Infrared Technology, 36(03), 2014, pp. 210-214.

24. XC Zhao., "MATLAB image processing: capability enhancement and application cases". Beijing: BeiHang University Press, China. 2014.

25. Ji-Bo Z, Guo-Fu P, Wei-Feng D., "Bottom track method in sidescan sonar data processing based on LOG operator". Marine Science Bulletin, 29(3), 2010, pp.324-328.

26. Chavez JR P S, Isbrecht J A, Galanis P., "Processing, mosaic king and management of the Monterey Bay digital sidescan -sonar images". Marine Geology, 181(1-3), 2002, pp.305-315.

27. Berkson J M, Clay C S., "Transformation of side-scan sonar records to a linear display". The International Hydrographic Review, 50(2), 2015, pp.55-59. 